\title{
Critical Current of Type-II Superconductors in a Broken Bose Glass State
}

\author{
J. P. Rodriguez ${ }^{1}$ \\ ${ }^{1}$ Department of Physics and Astronomy, \\ California State University, Los Angeles, California 90032
}

(Dated: November 1, 2018)

\begin{abstract}
The tilt modulus of a defective Abrikosov vortex lattice pinned by material line defects is computed using the boson analogy. It tends to infinity at long wavelength, which yields a Bose glass state that is robust to the addition of weak point-pinning centers, and which implies a restoring force per vortex line for rigid translations about mechanical equilibrium that is independent of magnetic field. It also indicates that the Bose glass state breaks into pieces along the direction of the correlated pinning centers if the latter have finite length. The critical current is predicted to crossover from two dimensional to three dimensional behavior as a function of sample thickness along the correlated pinning centers in such case. That crossover notably can occur at a film thickness that is much larger than that expected from point pins of comparable strength. The above is compared to the dependence on thickness shown by the critical current in certain films of high-temperature superconductors currently being developed for wire technology.
\end{abstract}




\section{INTRODUCTION}

It well known that thin films of high-temperature superconductors exhibit larger critical currents than their single-crystal counterparts. Thin films of the high-temperature superconductor $\mathrm{YBa}_{2} \mathrm{Cu}_{3} \mathrm{O}_{7-\delta}$ (YBCO) grown by pulsed laser deposition (PLD), which are actively being developed for wire technology, achieve critical currents that are a significant fraction of the maximum depairing current, for example[1]. Evidence exists that lines of dislocations that run parallel to the crystalline $c$ axis in PLD-YBCO act as correlated pinning centers for vortex lines inside of the superconducting state[2], and thereby give rise to such high critical currents. This is confirmed by the peak observed in the critical current of PLD-YBCO at orientations of the $c$ axis aligned parallel to an applied magnetic field, as well as by the dramatic enhancement of the former peak after more material defects in the form of nano-rods aligned parallel to the $c$ axis are added[3] [4].

The microstructure described above for PLD-YBCO films immediately suggests that the vortex lattice that emerges from the superconducting state in applied magnetic field aligned parallel to the $c$ axis is some form of Bose glass characterized by a divergent tilt modulus[5]. In the limit of a rigid vortex lattice, to be expected at large magnetic fields, two-dimensional (2D) collective pinning of vortex lines by the material line defects then determines the critical current [6] [7] [8] [9]. Recent theoretical calculations that follow this line of reasoning find moderate quantitative agreement with the critical current measured in films of PLD-YBCO in c-axis magnetic fields of a few to many $\mathrm{kG}$, at liquid nitrogen temperature[10]. A potential problem with the Bose glass hypothesis, however, is that the material line defects in PLD-YBCO films can meander, or they can be of relatively short length [3] [4]. That question is addressed in this paper, where we find that the Bose glass breaks up into pieces along the direction of the correlated pinning centers when the effective length of the latter is less than the film thickness. In particular, the profile of the critical current versus film thickness is predicted to reflect a crossover from two-dimensional to threedimensional (3D) collective pinning of the vortex lines[11]. This cross-over can occur at a length scale that is notably much larger than that expected from point pins of comparable strength[12]. We also find that the unbroken Bose glass state is robust to the addition of weak point pins. Good agreement is achieved between the dependence on thickness shown by the critical current in certain films of PLD-YBCO at applied magnetic field[12] and that 
predicted for the true Bose glass state[10]. Last, the effective restoring force per vortex line due to a rigid translation of the Bose glass about mechanical equilibrium, which is gauged by the Labusch parameter[13], is found to depend only weakly on applied magnetic field. This prediction agrees with recent measurements of the microwave surface resistance on PLD-YBCO films with nano-rod inclusions [14].

\section{TILT MODULUS OF BOSE GLASS}

Material line defects in thin enough films of PLD-YBCO can be considered to be perfectly parallel to the $c$-axis. They notably arrange themselves in a manner that resembles a snapshot of a 2D liquid[2], as opposed to a gas[15] [16]. In particular, such correlated pinning centers do not show clusters or voids. A defective vortex lattice that assumes a hexatic Bose glass state will then occur for external magnetic fields aligned in parallel to such a microstructure, in the limit of weak correlated pinning. It is characterized by parallel lines of edge dislocation defects that are injected into the pristine vortex lattice in order to relieve shear stress due to the correlated pins. The former do not show any intrinsic tendency to arrange themselves into grain boundaries, however, due to the absence of clusters and voids in the "liquid" arrangement of linear pinning centers. (Cf. refs. [15] and [16].) This results in a vortex lattice whose translational order is destroyed at long range by the isolated lines of edge dislocations, but which retains long-range orientational order. Collective pinning of the dislocation defects by the correlated pins then results both in an elastic response to shear and in a net superfluid density. Theoretical calculations[17] and numerical Monte Carlo simulations (see fig. 1 and caption) of the corresponding 2D Coulomb gas ensemble[18] confirm this picture.

The correlation length $L_{c}(\mid)$ for order along the magnetic field direction is infinite for a Bose glass state. In the limit of weak correlated pins, the density of dislocation defects that thread the corresponding vortex lattice can then be obtained by applying the theory of 2D collective pinning [6] [7] [8]. Each line of unbound edge dislocations is in one-to-one correspondence with a well-ordered bundle of vortex lattice, or Larkin domain, that has dimensions $R_{c}(\mid) \times R_{c}(\mid)$ in the directions transverse to the magnetic field. The injection of the dislocation lines into the pristine vortex lattice then results in plastic creep of each

Larkin domain by a Burgers vector[19]. The transverse Larkin scale is hence obtained by 
minimizing the sum of the elastic energy cost due to the edge dislocations with the gain in pinning energy due to the translation of a Larkin domain by an elementary Burgers vector of the triangular vortex lattice, $b=a_{\triangle}$. This yields the estimate[6] [7] [8]

$$
R_{c}(\mid)^{-2}=C_{0}^{2} n_{\mathrm{p}}\left(f_{\mathrm{p}} / c_{66} b\right)^{2},
$$

for the density of Larkin domains, which coincides with the density of lines of unbound dislocations. Here $n_{\mathrm{p}}$ denotes the density of pinned vortex lines, $f_{\mathrm{p}}$ denotes the maximum pinning force along a material line defect per unit length, and $c_{66}$ denotes the elastic shear modulus of the pristine vortex lattice. The prefactor above is of order [8] $C_{0} \cong \pi / \ln \left(R_{c} / a_{\mathrm{df}}^{\prime}\right)^{2}$, where $a_{\mathrm{df}}^{\prime}$ is the core diameter of a dislocation in the vortex lattice. Consider now the limit of weak pinning centers that do not crowd together: $f_{\mathrm{p}} \rightarrow 0$ and $\pi r_{\mathrm{p}}^{2} \cdot n_{\phi} \ll 1$, respectively, where $n_{\phi}$ denotes the density of material line defects, and where $r_{\mathrm{p}}$ denotes their range. Simple considerations of probability then yield the identity $n_{\mathrm{p}} / n_{\phi}=n_{\mathrm{B}} \cdot \pi r_{\mathrm{p}}^{2}$ between the fraction of occupied pinning centers and the product of the density of vortex lines, $n_{\mathrm{B}}$, with the effective area of each pinning center. Substituting it plus the estimate $c_{66}=\left(\Phi_{0} / 8 \pi \lambda_{L}\right)^{2} n_{\mathrm{B}}$ for the shear modulus of the pristine vortex lattice[21] into Eq. (1) then yields the result $R_{c}(\mid)^{-2} \cong(\sqrt{3} \pi / 2) C_{0}^{2}\left(4 f_{\mathrm{p}} r_{\mathrm{p}} / \varepsilon_{0}\right)^{2} n_{\phi}$ for the density of Larkin domains, which depends only weakly on magnetic field. Here, $\lambda_{L}$ denotes the London penetration depth and $\varepsilon_{0}=\left(\Phi_{0} / 4 \pi \lambda_{L}\right)^{2}$ is the maximum tension of a flux line in the superconductor. All of the above is valid in the $2 \mathrm{D}$ collective pinning regime that exists at perpendicular magnetic fields beyond the threshold $B_{\mathrm{cp}}=C_{0}^{2}(\sqrt{3} / 2)\left(4 f_{\mathrm{p}} / \varepsilon_{0}\right)^{2} \Phi_{0}$, in which case many vortex lines are pinned by material line defects within a Larkin domain of transverse dimensions $R_{c}(\mid) \times R_{c}(\mid)$ [10].

We will now exploit the boson analogy for vortex matter in order to compute the uniform tilt modulus of the hexatic Bose glass in the absence of point pinning centers [5]. It amounts to a London model set by the free-energy density

$$
g_{\mathrm{B}}(\{\mathbf{r}\}, z)=\sum_{i} \frac{1}{2} \tilde{\varepsilon}_{l}\left(\frac{d \mathbf{r}_{i}}{d z}\right)^{2}+\sum_{i>j} V_{0}\left(\mathbf{r}_{i}, \mathbf{r}_{j}\right)+\sum_{i} V_{\mathrm{p}}\left(\mathbf{r}_{i}\right)
$$

for vortex lines located at transverse positions $\left\{\mathbf{r}_{i}(z)\right\}$, at a coordinate $z$ along the field direction. Here $\tilde{\varepsilon}_{l}$ denotes the tension of an isolated vortex line, while the pair potential $V_{0}\left(\mathbf{r}, \mathbf{r}^{\prime}\right)$ describes the interaction between vortex lines at the same longitudinal coordinate $z$. The energy landscape for the correlated pinning centers is described by the potential 
energy $V_{\mathrm{p}}(\mathbf{r})$, which is independent of the coordinate $z$ along the field direction. The thermodynamics of this system in the presence of an external tilt stress $n_{\mathrm{B}} \mathbf{a}$ is then set by the partition function

$$
Z_{\mathrm{B}}[\mathbf{a}]=\left(\Pi_{i} \int \mathcal{D}\left[\mathbf{r}_{i}(z)\right]\right) \exp \left[-\left(k_{B} T\right)^{-1} \int_{0}^{L_{z}} d z\left[g_{\mathrm{B}}(\{\mathbf{r}\}, z)-\int d^{2} r \mathbf{j}_{\mathrm{B}} \cdot \mathbf{a}\right]\right]
$$

which under periodic boundary conditions, $\mathbf{r}_{i}\left(z+L_{z}\right)=\mathbf{r}_{i}(z)$, is equivalent to a system of $2 \mathrm{D}$ bosons. Above, $\mathbf{j}_{\mathrm{B}}(\mathbf{r}, z)=\sum_{i} \delta^{(2)}\left[\mathbf{r}-\mathbf{r}_{i}(z)\right]\left(d \mathbf{r}_{i} / d z\right)$ is the current density within the boson analogy. Observe now that the kernel $\Pi_{\mu, \nu}(\omega)$ of the uniform electromagnetic response for alternating current $(\mathrm{AC})$ is connected to this partition function through the proportionality relationship

$$
Z_{\mathrm{B}}[\mathbf{a}] \propto \exp \left[\left(k_{\mathrm{B}} T\right)^{-1} V \sum_{i \omega_{n}} \frac{1}{2} \mathbf{a} \cdot \boldsymbol{\Pi} \cdot \mathbf{a}\right]
$$

in the limit that the corresponding uniform tilt stress vanishes, $\mathbf{a} \rightarrow 0$. Above, the Matsubara frequencies $i \omega_{n}$ are given by the allowed wavenumbers along the magnetic field, $q_{z}=2 \pi n / L_{z}$, and $V=L_{x} L_{y} L_{z}$ is the volume of the system. The fact that the tilt stress is given by $n_{\mathrm{B}} \mathbf{a}$ then yields the identity

$$
C_{44}\left(q_{z}\right)=n_{\mathrm{B}}^{2} / \Pi_{\perp}\left(\omega_{n}\right)
$$

between the uniform tilt modulus and the uniform AC electromagnetic response of the 2D Bose glass. The subscript " $\perp$ " above is a tag for the pure shear component of the electromagnetic kernel $\Pi_{\mu, \nu}$ (Cf. ref. [5] ).

The hexatic Bose glass state is clearly a 2D dielectric insulator within the boson analogy. Its electromagnetic response can therefore be modeled by the kernel

$$
\Pi_{\perp}(\omega)=\left(n_{\mathrm{B}} / \tilde{\varepsilon}_{l}\right) \omega^{2} /\left(\omega^{2}-\omega_{0}^{2}\right),
$$

which is dielectric in the low-frequency limit, and which conserves charge by satisfying the oscillator f-sum rule. Above, $\omega_{0}$ is the natural frequency of the electric dipole degrees of freedom in the Bose glass. The latter correspond to the 2D Larkin domains in reality, since they represent the smallest units of well-ordered vortex lattice that can respond independently to an applied force. We then have that the above natural frequency is of order the resonant frequency for transverse sound inside a Larkin domain of the 2D Bose glass: $\omega_{0} \sim \gamma / R_{c}(\mid)$, where $\gamma=\left(c_{66} / c_{44}\right)^{1 / 2}$ is the effective mass anisotropy parameter equal to the transverse sound speed within the boson analogy. Here $c_{44}=n_{\mathrm{B}} \tilde{\varepsilon}_{l}$ is the tilt modulus due to isolated 
flux lines. After substitution into Eqs. (6) for the AC response, the identity (5) then yields a divergent tilt modulus for the Bose glass at long wavelength

$$
C_{44}\left(q_{z}\right)=n_{\mathrm{B}} \tilde{\varepsilon}_{l}\left[1+\left(q_{z} L_{*}\right)^{-2}\right]
$$

with a longitudinal scale $L_{*}=\omega_{0}^{-1}$ that is related to the transverse Larkin length by the anisotropic scale transformation $L_{*} \sim R_{c}(\mid) / \gamma$.

Expression (7) for the uniform tilt modulus of a Bose glass is the central result of the paper. We shall first extract the Labusch parameter[13] from the singular behavior that it shows at long wavelength. In particular, observe that the elastic energy density for a periodic tilt of the Bose glass by a displacement $u_{0}$ at long wavelength, $\frac{1}{2} C_{44}\left(q_{z}\right) q_{z}^{2} u_{0}^{2}$, acquires a contribution of the form $\frac{1}{2} k_{0} u_{0}^{2}$ from this divergence, with $k_{0}=c_{66} / R_{c}(\mid)^{2}$. The latter is simply the spring constant per unit volume of the restoring force for a rigid translation of the Bose glass state about mechanical equilibrium. Using the estimate $c_{66}=\frac{1}{4} \varepsilon_{0} n_{\mathrm{B}}$ for the shear modulus of the vortex lattice[21] yields an effective spring constant per vortex line due to $2 \mathrm{D}$ collective pinning limited by plastic creep, $k_{0} / n_{\mathrm{B}}$, that is given by $k_{\mathrm{p}}=\frac{1}{4} \varepsilon_{0} / R_{c}(\mid)^{2}$. Notice that $k_{\mathrm{p}}$ depends only weakly on magnetic field. The Labusch parameter extracted from the microwave surface resistance on PLD-YBCO films with nano-rod inclusions also exhibits only a weak dependence on external magnetic field aligned parallel to the nano-rods (or c-axis) [14]! The above should be compared to the corresponding Labusch parameter due to point pins[13], which depends strongly on magnetic field. Indeed, given the conjecture $k_{\mathrm{p}}=\left(c_{66} / R_{c}^{2}+c_{44} / L_{c}^{2}\right) / n_{\mathrm{B}}$ for the Labusch parameter due to $3 \mathrm{D}$ collective-pinning implies that it decays with increasing magnetic field instead as $1 / B^{2}$ in such case. Here we have assumed anisotropic scaling, and we have used[9] $R_{c} \propto B$.

We shall next use Eq. (77) for the uniform tilt modulus to test how robust the hexatic Bose glass is to the addition of point pinning centers. The hexatic Bose glass shows long-range orientational order in all directions (see fig. 1). The addition of point pins will therefore break it up into Larkin domains, of dimensions $R_{c}^{\prime} \times R_{c}^{\prime} \times L_{c}$ transverse and parallel to the magnetic induction, that tilt in the transverse direction by a distance of order the size of the vortex core, $\xi$. Such a break-up then has an elastic energy cost per unit volume and a pinning energy gain per unit volume that sum to[6] [9]

$$
\delta u=\frac{1}{2} C_{66}\left(\frac{\xi}{R_{c}^{\prime}}\right)^{2}+\frac{1}{2} C_{44}\left(\frac{\xi}{L_{c}}\right)^{2}-\left(\frac{n_{0}^{\prime}}{R_{c}^{\prime 2} L_{c}}\right)^{1 / 2} f_{0}^{\prime} \xi .
$$


Here $C_{66}$ denotes the shear modulus of the hexatic Bose glass, which can be approximated by $c_{66}$ in the limit of weak correlated pinning, while the corresponding tilt modulus $C_{44}$ is given by expression (17) evaluated at wavenumber $q_{z}=1 / L_{c}$. Also, $n_{0}^{\prime}$ denotes the density of point pins, while $f_{0}^{\prime}$ denotes the magnitude of their characteristic force. Minimizing $\delta u$ with respect to the dimensions of the Larkin domains then yields standard results for these[9]: $L_{c}=L_{c}(\cdot)\left[1+\left(q_{z} L_{*}\right)^{-2}\right]$ and $R_{c}^{\prime}=R_{c}(\cdot)\left[1+\left(q_{z} L_{*}\right)^{-2}\right]^{1 / 2}$, where $L_{c}(\cdot)=2 c_{44} c_{66} \xi^{2} / n_{0}^{\prime} f_{0}^{\prime 2}$ and $R_{c}(\cdot)=2^{1 / 2} c_{44}^{1 / 2} c_{66}^{3 / 2} \xi^{2} / n_{0}^{\prime} f_{0}^{\prime 2}$ are respectively the longitudinal Larkin scale and the transverse Larkin scale in the absence of correlated pinning centers. The first equation is quadratic in terms of the variable $L_{c}^{-1}$, and it has a formal solution $L_{c}^{-1}=\left(2 L_{c}(\cdot)\right)^{-1}+\left[\left(2 L_{c}(\cdot)\right)^{-2}-L_{*}^{-2}\right]^{1 / 2}$. The hexatic Bose glass is therefore robust to the addition of weak point pins. In particular, the longitudinal Larkin scale $L_{c}$ remains divergent for $L_{c}(\cdot)>L_{*} / 2$, which is equivalent to the inequality $2^{3 / 2} R_{c}(\cdot)>R_{c}(\mid)$.

\section{D-3D CROSSOVER IN CRITICAL CURRENT}

The critical current density of the above hexatic Bose glass, which is robust to the addition of point pins, can be obtained by applying $2 \mathrm{D}$ collective pinning theory[10]. All vortex lines can be considered to be rigid rods. Balancing the Lorentz force against the collective pinning force over a Larkin domain [6] [9] yields the identity $J_{c} B / c=\left[\left(n_{\mathrm{p}} f_{\mathrm{p}}^{2}+n_{\mathrm{p}}^{\prime} f_{\mathrm{p}}^{\prime 2}\right) / R_{c}^{2}\right]^{1 / 2}$ for the product of the critical current density along the film, $J_{c}$, with the perpendicular magnetic field, $B$, aligned parallel to the material line defects[10]. Here $n_{\mathrm{p}}$ and $n_{\mathrm{p}}^{\prime}$ denote the density of vortex lines pinned by material line defects and the density of interstitial vortex lines pinned by material point defects, while $f_{\mathrm{p}}$ and $f_{\mathrm{p}}^{\prime}$ are the maximum force exerted on the respective vortex lines per unit length. Again, it is important to observe that the critical current is limited by plastic creep of the vortex lattice due to slip of the quenched-in lines of edge dislocations along their respective glide planes[19]. Minimization of the sum of the elastic and pinning energy densities then yields a higher density of Larkin domains in the hexatic Bose glass with material point defects added by comparison (1) : $R_{c}^{-2}=C_{0}^{2}\left(n_{\mathrm{p}} f_{\mathrm{p}}^{2}+n_{\mathrm{p}}^{\prime} f_{\mathrm{p}}^{\prime 2}\right) /\left(c_{66} b\right)^{2}$. This reflects the injection of extra lines of edge dislocations that relieve shear stress caused by point pins. Substitution in turn yields a critical current density, $J_{c}=j_{c}+j_{c}^{\prime}$, that has a component due to correlated pins set by the identity

$$
j_{c} B / c=C_{0} n_{\mathrm{p}} f_{\mathrm{p}}^{2} / c_{66} b
$$


and that has a component due to point pins set by the ratio $j_{c}^{\prime} / j_{c}=n_{\mathrm{p}}^{\prime} f_{\mathrm{p}}^{\prime 2} / n_{\mathrm{p}} f_{\mathrm{p}}^{2}$. The critical current density notably varies as $J_{c} \propto B^{-1 / 2}$ with magnetic field in the limit of weak pinning[10].

Consider now a film geometry of thickness $\tau$ along the axis of the material line defects. The forces due to point pins add up statistically along a rigid interstitial vortex line. The effective pinning force per unit length experienced by an interstitial vortex line is then given by [7] $f_{\mathrm{p}}^{\prime}=f_{0}^{\prime} /\left(\tau_{\mathrm{p}}^{\prime} \tau\right)^{1 / 2}$ at film thicknesses $\tau$ that are much greater than the average separation $\tau_{\mathrm{p}}^{\prime}$ between such pins along the field direction. Again, $f_{0}^{\prime}$ denotes the maximum force exerted by a point pin. The relative contribution by point pins to the critical current density is then predicted to show an inverse dependence on film thickness, $j_{c}^{\prime} / j_{c}=\tau_{0} / \tau$, that is set by the scale $\tau_{0}=\left(n_{\mathrm{p}}^{\prime} / n_{\mathrm{p}}\right)\left(f_{0}^{\prime 2} / f_{\mathrm{p}}^{2} \tau_{\mathrm{p}}^{\prime}\right)$. We thereby obtain the linear dependence on film thickness $I_{c}^{(2 D)}=\left(\tau_{0}+\tau\right) j_{c}$ for the net critical current per unit width, $\tau J_{c}$. Last, comparison of Eq. (11) with Eq. (9) yields the useful expression

$$
L_{*}=\gamma^{-1}\left[\left(3^{5 / 4} / 2^{7 / 2} C_{0}\right)\left(\xi \cdot a_{\mathrm{vx}}\right)\left(j_{0} / j_{c}\right)\right]^{1 / 2}
$$

for the longitudinal scale characteristic of the Bose glass as a function of the bulk critical current density, $j_{c}$. Here $a_{\mathrm{vx}}=n_{\mathrm{B}}^{-1 / 2}$ is the average distance between vortex lines and $j_{0}$ is the depairing current density (see ref. [9]). Figure 2 shows a fit to data for the critical current versus thickness obtained from a thin film of PLD-YBCO at liquid-nitrogen temperature in $1 \mathrm{~T}$ magnetic field aligned parallel to the $c$ axis[12]. A bulk critical current density $j_{c}=0.22 \mathrm{MA} / \mathrm{cm}^{2}$ is extracted from it. Using a value of $j_{0}=36 \mathrm{MA} / \mathrm{cm}^{2}$ for the depairing current (ref. [12]), of $\xi=11 \mathrm{~nm}$ for the coherence length, of $\gamma=7$ for the mass anisotropy parameter, and setting $C_{0}=1$ yields a longitudinal scale $L_{*}=24 \mathrm{~nm}$.

Finally, consider again an arrangement of material line defects that are aligned along the $c$ axis, that show no voids or clusters in the transverse directions, but that are broken up into relatively long rods of length $L_{0} \gg L_{*}$. It can be realized by meandering material line defects [1] [2], where $L_{0}$ is the correlation length for alignment along the $c$-axis, or by artificial nano-rod defects[3] [4]. Expression (7) for the divergent tilt modulus indicates proximity to the regime of $2 \mathrm{D}$ collective pinning, where both $L_{c}$ and $L_{0}$ are divergent. It hence indicates that $L_{c}$ is also large compared to $L_{*}$ here. Second, observe that $L_{*} \sim R_{c}(\mid) / \gamma$ coincides with the longitudinal Larkin scale if the rods are considered to be point defects. The previous ultimately implies the chain of inequalities $L_{0} \geq L_{c} \gg L_{*}$. They are consistent 
with a broken Bose glass state for the vortex lattice, which is threaded by isolated lines of edge dislocations of length $L_{c}$ along the $c$-axis that are connected together by lines of screw-dislocations [19] along the transverse directions[11]. As in the limiting case of the true Bose glass state (fig. 1), the lines of edge dislocations are injected into the pristine vortex lattice in order to relieve shear stress due to the correlated pins. Larkin domains hence are finite volumes [6] [9]. In contrast to their transverse dimensions, however, Larkin domains exhibit well defined boundaries along the direction parallel to the correlated pinning centers, across which the vortex lattice slips by a Burger's vector due to the presence of the screw dislocations[19]. The critical current density expected from this peculiar example of 3D collective pinning therefore coincides with that of a Bose glass of thickness $L_{c}: J_{c}=j_{c}+j_{c}^{\prime}$, with a bulk component due to interstitial vortex lines $j_{c}^{\prime} / j_{c}=\tau_{0} / L_{c}$. The critical current per unit width then varies with film thickness as $I_{c}^{(3 D)}=\tau J_{c}$, showing no offset. Figure 2 depicts the predicted dependence on film thickness for the critical current of such a broken Bose glass. It notably exhibits 2D-3D cross-over at film thicknesses in the vicinity of $L_{c}$ [11].

\section{CONCLUDING REMARKS}

The dependence of the critical current on thickness $\tau$ shown by certain films of PLDYBCO is in fact consistent with dimensional cross-over at $\tau \sim 1 \mu \mathrm{m}$. A previous attempt by Gurevich to account for such behavior by collective pinning of individual vortex lines at point defects yields a longitudinal Larkin scale $L_{c} \sim 10 \mathrm{~nm}$ that is too small, however[12]. We find here, on the other hand, that pinning due to correlated material defects of length $L_{0}$ yields a Larkin scale $L_{c}$ that is much longer than that $\left[L_{*}=24 \mathrm{~nm}\right.$, see Eq. (10)] expected from point pins of comparable strength if $L_{0}$ is much longer than that scale as well. Indeed, we predict here that the film thickness at which the critical current crosses over from 2D to $3 \mathrm{D}$ behavior is of order the effective length of the correlated pinning centers when that length satisfies $L_{0} \gg L_{*}$.

The author thanks Leonardo Civale, Chandan Das Gupta and Sang-il Kim for discussions. This work was supported in part by the US Air Force Office of Scientific Research under grant no. FA9550-06-1-0479. 
[1] B. Dam, J.M. Huijbregtse, F.C. Klaassen, R.C.F. van der Geest, G. Doornbos, J.H. Rector, A.M. Testa, S. Freisem, J.C. Martinez, B. Stauble-Pumpin and R. Griessen, Nature 399, 439 (1999).

[2] F.C. Klaassen, G. Doornbos, J.M. Huijbregtse, R.C.F. van der Geest, B. Dam and R. Griessen, Phys. Rev. B 64, 184523 (2001).

[3] J.L. MacManus-Driscoll, S.R. Foltyn, Q.X. Jia, H. Wang, A. Serquis, L. Civale, B. Maiorov, M.E. Hawley, M.P. Maley and D.E. Peterson, Nature Materials 3, 439 (2004).

[4] A. Goyal, S. Kang, K.J. Leonard, P.M. Martin, A.A. Gapud, M. Varela, M. Paranthaman, A.O. Ijaduola, E.D. Specht, J.R. Thompson, D.K. Christen, S.J. Pennycook and F.A List, Supercond. Sci. Technol. 18, 1533 (2005).

[5] D.R. Nelson and V.M. Vinokur, Phys. Rev. B 48, 13060 (1993).

[6] A.I. Larkin and Yu V. Ovchinnikov, J. Low Temp. Phys. 34, 409 (1979).

[7] P.H. Kes and C.C. Tsuei, Phys. Rev. B 28, 5126 (1983).

[8] S.J. Mullock and J.E. Evetts, J. Appl. Phys. 57, 2588 (1985).

[9] M. Tinkham, Introduction to Superconductivity (McGraw-Hill, New York, 1996) 2nd ed..

[10] J.P. Rodriguez and M.P. Maley, Phys. Rev. B 73, 094502 (2006).

[11] R. Wördenweber and P. H. Kes, Phys. Rev. B 34, 494 (1986).

[12] Chang-Beom Eom, Alex Gurevich and David Larbalestier, Superconductivity for Electric Systems 2004 Annual (DOE) Peer Review (http://www.energetics.com/supercon04.html).

[13] G. Blatter, V.B. Geshkenbein, and J.A.G. Koopmann, Phys. Rev. Lett. 92, 067009 (2004).

[14] N. Pompeo, R. Rogai, E. Silva, A. Augieri, V. Galluzzi and G. Celentano, Appl. Phys. Lett. 91, 182507 (2007). (arXiv:0710.1754).

[15] M. Menghini, Y. Fasano, F. de la Cruz, S.S. Banerjee, Y. Myasoedov, E. Zeldov, C.J. van der Beek, M. Konczykowski, and T. Tamegai, Phys. Rev. Lett. 90, 147001 (2003).

[16] C. Dasgupta and O.T. Valls, Phys. Rev. Lett. 91, 127002 (2003).

[17] J.P. Rodriguez, Phys. Rev. B 72, 214503 (2005).

[18] C.E. Creffield and J.P. Rodriguez, Phys. Rev. B 67, 144510 (2003).

[19] D. Hull and D.J. Bacon, Introduction to Dislocations, 3rd ed. (Pergamon, Oxford, 1984).

[20] J.P. Rodriguez, Phys. Rev. B 70, 224507 (2004). 
[21] E.H. Brandt, J. Low Temp. Phys. 26, 735 (1977). 


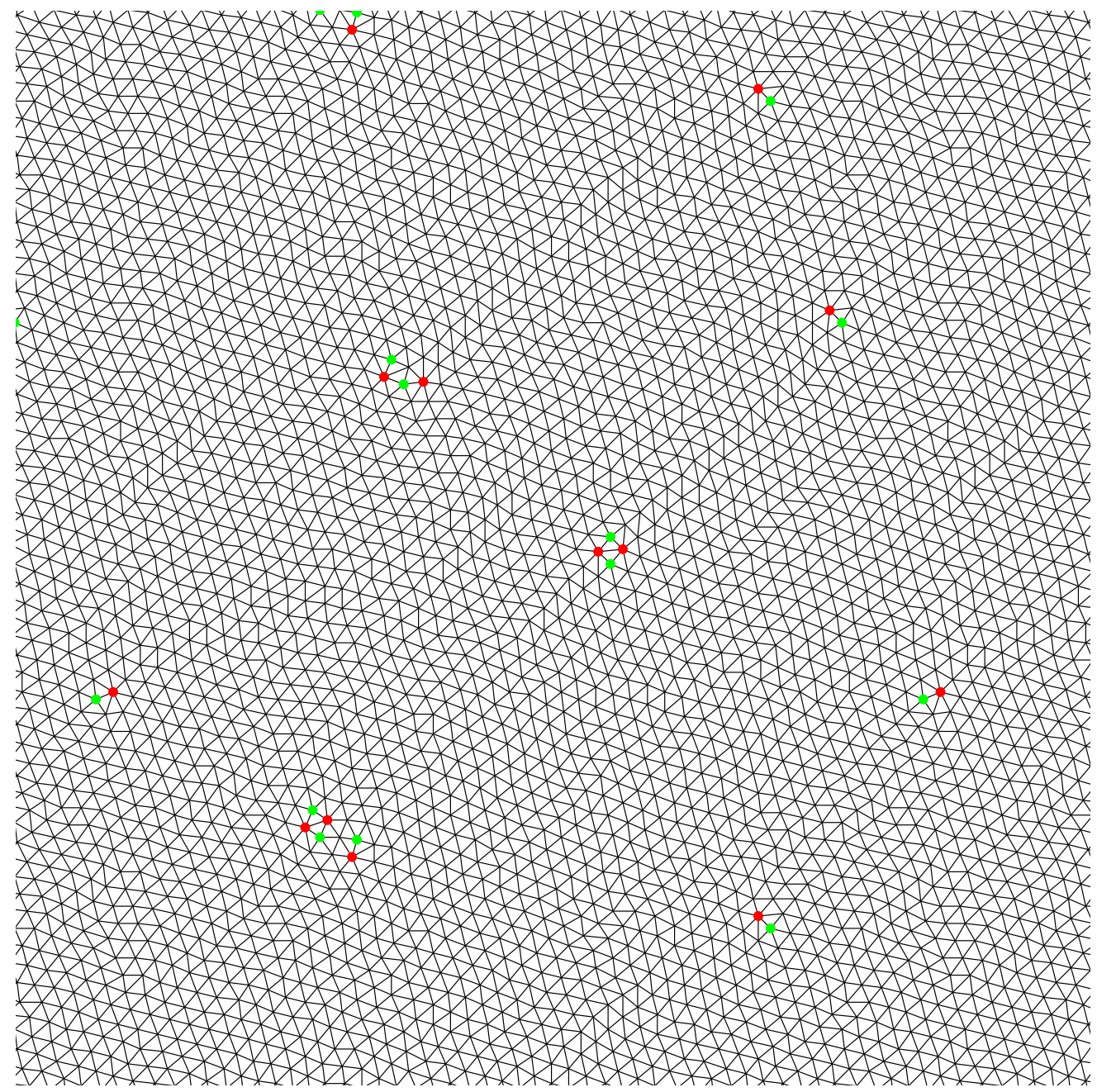

FIG. 1: Shown is a Delaunay triangulation of a low-temperature groundstate made up of 2016 vortices that interact logarithmically over a $336 \times 336$ grid with periodic boundary conditions, and that experience an equal number of identically weak $\delta$-function pinning centers arranged in a "liquid" fashion. (See ref. [18].) The state shows macroscopic phase coherence and long-range hexatic order, with a supefluid density and a hexatic order parameter, respectively, that are $29 \%$ and $58 \%$ of the maximum possible values attained by the perfect triangular vortex lattice. It was obtained after simulated annealing from the liquid state down to low temperature, which resulted in 371 pinned vortices. A red and green pair of disclinations forms a dislocation (see ref. [19]). The above Monte Carlo simulation results are consistent with theoretical predictions of a hexatic vortex glass state in two dimensions, in the zero-temperature limit, for pinning arrangements that do not show any clusters or voids (ref. [17]). Josephson coupling between layers then produces a Bose glass transition above zero temperature as long as the 2D glass transtion is second order (ref. [20]). 


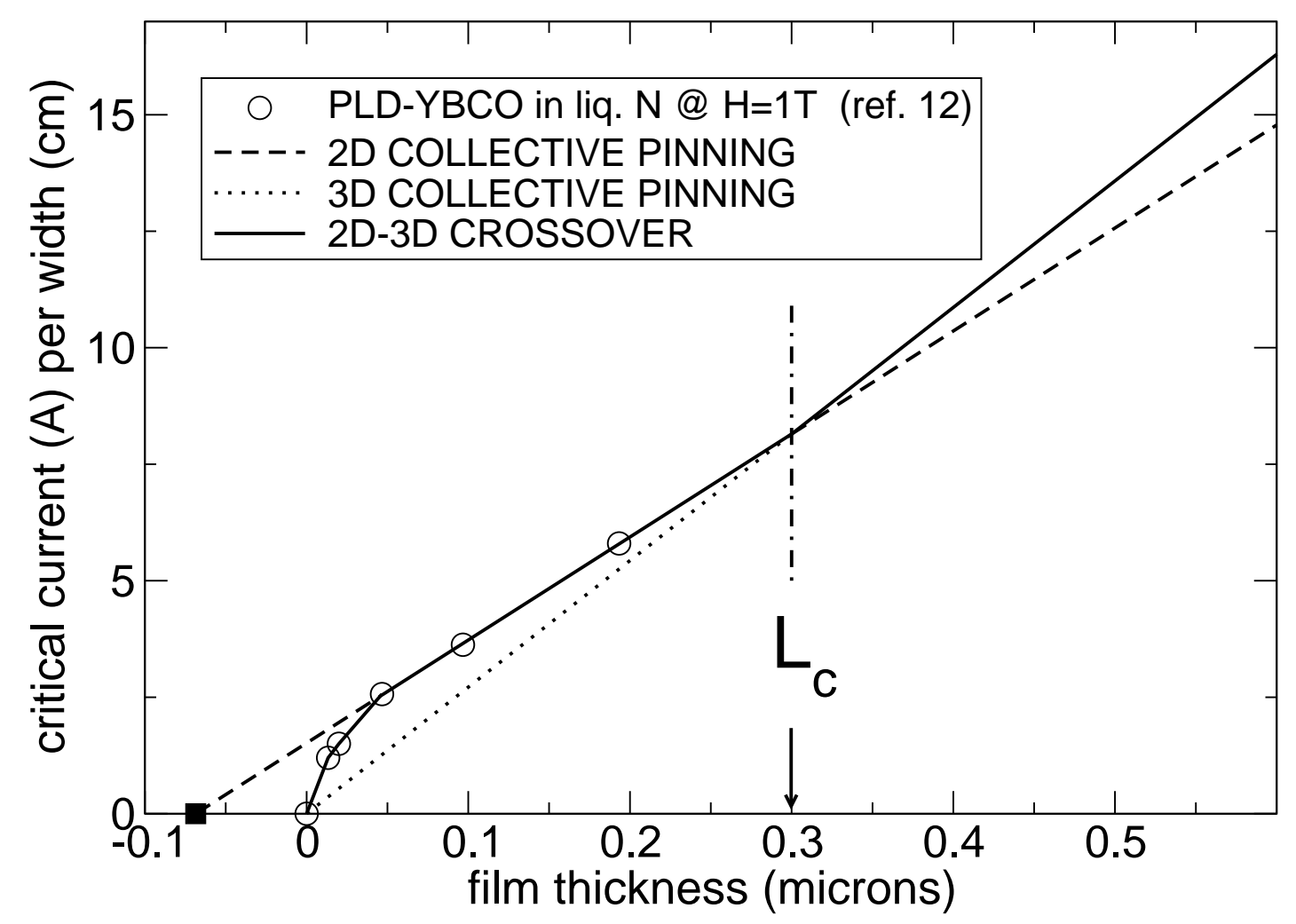

FIG. 2: Shown is the dependence on film thickness predicted for the critical current of a defective vortex lattice found in a broken Bose glass state (solid line). The dashed and dotted lines are extrapolated from the 2D and 3D behaviors, respectively. Measurements made by Sang Kim (circles, ref. [12]) of the critical current on a thin film of PLD-YBCO at liquid nitrogen temperature subject to $1 \mathrm{~T}$ magnetic field aligned parallel to the c-axis are fit to the straight dashed line predicted by $2 \mathrm{D}$ collective pinning (ref. [10]). This yields an intercept $-\tau_{0}=-69 \mathrm{~nm}$ and a slope $j_{c}=0.22 \mathrm{MA} / \mathrm{cm}^{2}$. Although the value of $L_{c}$ shown here is indeed larger than the lower bound $L_{*}=24 \mathrm{~nm}$ [see Eq. (10)] and is consistent with the fit to $2 \mathrm{D}$ collective pinning, it is only hypothetical. 